\title{
O centro instantâneo de movimento e vetor velocidade em joelhos submetidos a incisuroplastia troclear e estabilização articular após transecção do ligamento cruzado cranial em cães
}

\author{
Instantaneous center of motion and velocity vector in stifle of dogs undergoing intercondylar \\ notchplasty and articular repair following transection of the cranial cruciate ligament
}

\author{
André Luis Selmi I João Guilherme Padilha FilhoII Bruno Testoni Lins ${ }^{\text {III }}$ \\ Guilherme Maia Mendes ${ }^{\text {III }}$ Gisleine Cristina Eimantas ${ }^{\mathrm{I}}$
}

\section{RESUMO}

\begin{abstract}
Avaliou-se o centro instantâneo de movimento e vetor velocidade após a transecção do ligamento cruzado cranial (LCCr), seguida da substituição ligamentar por retalho de fáscia lata, associada ou não a incisuroplastia troclear (ITR) em nove cães adultos. O joelho direito (GI) foi submetido a ITR e posterior estabilização articular, e o joelho esquerdo submetido somente a substituição ligamentar (GC). Os animais foram avaliados nos períodos pré-operatório, pós-operatório imediato e aos 30, 90 e 180 de pós-operatório, correspondente ao momento de eutanásia de subgrupos de três animais. $O$ centro instantâneo de movimento (CIM) e o vetor velocidade $(V v)$ resultante foram determinados por meio do deslocamento de pontos a partir da análise radiográfica dos joelhos. Todas as articulações apresentavam CIM e Vv normais antes da transecção do ligamento cruzado cranial. Após a estabilização articular, acompanhada ou não de ITR, observou-se o posicionamento normal do CIM e Vv resultante, em todos os períodos de avaliação, apesar da presença de movimento de gaveta em três animais de GC e dois de GI no pós-operatório imediato, em dois animais de cada grupo, aos 30 dias de avaliação, e em um animal em cada grupo nas avaliações subseqüentes. Conclui-se que a estabilização articular com retalho de fáscia lata, associada ou não a ITR, mantém a integridade biomecânica do joelho, quando considerados o CIM e o $\mathrm{VV}$
\end{abstract}

Palavras-chave: biomecânica, radiografia, desmotomia.

\section{ABSTRACT}

The instantaneous center of motion (ICM) and velocity vector $(\mathrm{Vv})$, after transection of the cranial cruciate ligament (CCL) followed by a fascial strip reconstruction in association with intercondylar notchplasty (IN), were studied in nine adult dogs. The right stifle was submitted to IN followed by fascial strip reconstruction of the CCL (GI) while in the left stifle IN was not performed (GC). Dogs were evaluated the day prior to surgery, immediately after surgery and at 30, 90 and 180 days postoperatively (po), time at which a subgroup of three dogs were euthanatized. The ICM and resulting Vv were determined by radiographic examination of the stifle. All the stifles presented normally positioned ICM and Vv before surgery. No changes were observed in ICM or $V v$ in all stifles following intra-articular repair, in association or not with IN, throughout the evaluation period, despite the fact that three dogs in GC and two in GI presented a positive cranial drawer sign immediately following surgery, two dogs in GC and GI at 30 days po, and one single dog in each group thereafter until 180 days po. It is concluded that articular repair, in association with IN or not, did not alter stifle biomechanics, in respect to ICM and Vv.

Key words: biomechanics, radiography, desmotomy.

\section{INTRODUÇÃO}

A ruptura do ligamento cruzado cranial (RLCCr) é a causa mais freqüente de claudicação em membro pélvico diagnosticada na prática clínicocirúrgica de pequenos animais (PROSTREDNY et al., 1991; ROOSTER \& VAN BREE, 1999). Classicamente, a RLCCr é diagnosticada pela presença da instabilidade articular craniocaudal e posteriormente confirmada durante a intervenção cirúrgica (ARNOCZKY et al., 1977; SHIRES \& HULSE, 1984; COETZEE \& LUBBE, 1995), apesar de avaliações radiográficas com a articulação sob estresse serem recomendadas como auxílio diagnóstico (ROOSTER \& VAN BREE, 1999).

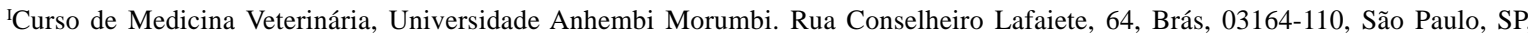
Brasil. E-mail:selmi@anhembi.br. Autor de correspondência.

IIDepartamento de Clínica e Cirurgia Veterinária, Faculdade de Ciências Agrárias e Veterinárias (FCAV), Universidade Estadual Paulista (UNESP). Jaboticabal, SP, Brasil.

IIICurso de Pós-graduação em Cirurgia Veterinária da Faculdade de Medicina Veterinária e Zootecnia (FMVZ), UNESP, Botucatu, SP, Brasil.
} 
Numerosas técnicas cirúrgicas têm sido descritas para a estabilização articular após a RLCCr, sendo tradicionalmente divididas em intra ou extraarticulares. As divergências em relação à superioridade das técnicas quanto ao melhor retorno da função e quanto à estabilidade articular são constantes, e alguns estudos têm sugerido que não são todos os procedimentos que resultam em biomecânica eficiente (ARNOCZKY et al., 1977; SELMI et al., 2003). Acreditase que os procedimentos de reconstrução intraarticular propiciem melhor posicionamento anatômico do material empregado na substituição do ligamento rompido, sendo mais indicados na manutenção da biomecânica articular por mimetizarem o trajeto do ligamento cruzado cranial (SHIRES \& HULSE, 1984; ELKINS et al., 1991; VASSEUR \& BERRY, 1992; COETZEE \& LUBBE, 1995; GEELS et al., 2000; SELMI et al., 2002). Dentre as várias técnicas intra-articulares empregadas na estabilização articular após a RLCCr em cães, a descrita por SHIRES \& HULSE (1984), conhecida como "under-and-over”, e posteriormente modificada por COETZEE \& LUBBE (1995), tem demonstrado resultados clínicos satisfatórios. Resumidamente, esta consiste no preparo de uma faixa de fáscia lata que se mantém inserida na tuberosidade da tíbia, conduzida através da fossa intercondilar por debaixo do ligamento intermeniscal, com emergência caudal sobre o côndilo lateral do fêmur, de onde é posteriormente tracionada e suturada ao tendão patelar. Apesar do grande número de estudos clínicos realizados utilizando técnicas intra-articulares (SHIRES \& HULSE, 1984; VASSEUR \& BERRY, 1992; COETZEE \& LUBBE, 1995; JEVENS et al., 1996; VASSEUR et al., 1996; GEELS et al., 2000; SELMI et al., 2002), a estenose da fossa intercondilar (EFI), decorrente da formação de osteófitos na fossa intercondilar secundária à instabilidade articular, tem recebido pouca atenção na reconstrução intra-articular da RLCCr em Medicina Veterinária(VASSEUR \& BERRY, 1992; AIKEN et al., 1995; FITCH et al., 1995a; FITCH et al., 1995b; VASSEUR et al., 1996). A EFI previne o posicionamento isométrico e ortotópico de substitutos ligamentares, além de predispor ao contato excessivo entre o neoligamento e a superfície articular do aspecto medial do côndilo lateral do fêmur, podendo resultar em ruptura precoce do enxerto (VASSEUR \& BERRY, 1992; AIKEN et al., 1995; FITCH et al. 1995b; VASSEUR et al., 1996; LA PRADE et al., 1998; MANN et al., 1999; SELMI et al., 2002). Por conseguinte, a realização de incisuroplastia troclear, que consiste no desgaste da fossa intercondilar estenosada, apesar de ser procedimento relativamente freqüente em humanos com RLCCr(LA PRADE et al., 1998; MANN et al., 1999), apresenta-se pobremente descrita em Medicina Veterinária (AIKEN et al., 1995; FITCH et al., 1995a; FITCH et al., 1995b).

Várias formas de avaliação pós-operatória têm sido utilizadas para verificar o resultado da estabilização cirúrgica após a RLCCr, incluindo a presença ou não do movimento de gaveta (SHIRES \& HULSE, 1984; PROSTREDNY et al., 1991; COETZEE \& LUBBE, 1995; LA PRADE et al., 1998; GEELS et al., 2000), a qualidade subjetiva de apoio do membro, o perímetro muscular da coxa e a amplitude de movimento articular (SHIRES \& HULSE, 1984; INNES \& BARR, 1998; LA PRADE et al., 1998), o uso de placa de força (JEVENS et al., 1996; GEELS et al., 2000), e avaliações radiográficas para documentação da progressão de osteoartrose (SHIRES \& HULSE, 1984; COETZEE \& LUBBE, 1995; GEELS et al., 2000) ou para determinação do centro instantâneo de movimento (CIM) (PROSTREDNY et al., 1991; SELMI et al., 2003).

O CIM é considerado como um ponto localizado no fêmur, em qualquer fase da amplitude de movimento deste osso em relação à tíbia, no qual a velocidade vetorial é igual a zero, e traduz-se pelo eixo de rotação articular num determinado momento submetido à neutralidade (IRELAND et al., 1986; PROSTREDNY et al., 1991), onde o movimento articular normal pode ser predominantemente de rolamento ou deslizamento, e representa uma forma simples e eficiente de se avaliar a biomecânica articular (MITTON et al., 1991; PROSTREDNY et al., 1991; SELMI et al., 2003).

O objetivo deste estudo foi analisar os efeitos da estabilização intra-articular quanto ao CIM e ao Vv após transecção do ligamento cruzado cranial, acompanhada ou não da incisuroplastia troclear, no joelho, em cães.

\section{MATERIAL E MÉTODOS}

Foram utilizados nove cães hígidos, sem raça definida, machos ou fêmeas, adultos, com massa

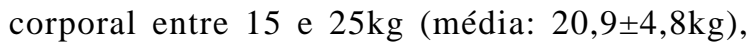
provenientes do Centro de Controle de Zoonoses. Os animais foram colocados em canis individuais com aproximadamente $1,5 \mathrm{~m}^{2}$ de área. Receberam ração comercial $^{\mathrm{a}}$ e água ad libitum.

Para o desenvolvimento deste estudo, os cães tiveram o joelho direito submetido a ITR e posterior estabilização articular (GI), enquanto que o joelho esquerdo foi submetido somente à substituição ligamentar (GC). Os cães foram agrupados em três subgrupos correspondentes ao período de eutanásia no $30^{\circ}, 90^{\circ}$ e $180^{\circ}$ dia pós-operatório. 
Para realização dos procedimentos cirúrgicos, os animais foram mantidos em jejum hídrico e alimentar nas 12 horas que antecederam o ato cirúrgico. A medicação pré-anestésica foi realizada com maleato de acepromazina ${ }^{\mathrm{b}}\left(0,1 \mathrm{mg} \mathrm{kg}^{-1}-\mathrm{IV}\right)$. A anestesia cirúrgica foi induzida, 15 minutos após, com propofol ${ }^{\mathrm{c}}$ $\left(6 \mathrm{mg} \mathrm{kg} \mathrm{kg}^{-1}-\mathrm{IV}\right)$. Os animais foram medicados com cetoprofeno $^{\mathrm{d}}$ ( $\left.2,2 \mathrm{mg} \mathrm{kg}^{-1}-\mathrm{IV}\right)$, e posicionados para realização de anestesia epidural empregando-se associação de sulfato de morfina ${ }^{\mathrm{e}}\left(0,1 \mathrm{mg} \mathrm{kg}^{-1}\right)$, cloridrato de lidocaína ${ }^{\mathrm{f}}\left(1,0 \mathrm{mg} \mathrm{kg}^{-1}\right)$ e de bupivacaína ${ }^{\mathrm{g}}\left(0,5 \mathrm{mg} \mathrm{kg}^{-1}\right)$. Os animais foram intubados e a anestesia mantida em circuito semifechado empregando-se sevoflurano ${ }^{\mathrm{h}}$ vaporizado em oxigênio. Em seguida, foi administrada cefalexina sódicai $\left(20 \mathrm{mg} \mathrm{kg}^{-1}-\mathrm{IV}\right)$. Durante todo o período anestésico, os animais receberam solução de Ringer com lactato (10 $\left.\mathrm{mL} \mathrm{kg}^{-1} \mathrm{hr}^{-1}-\mathrm{IV}\right)$.

Abordou-se a articulação a partir de incisão parapatelar lateral. Após identificação, secção e remoção do LCCr, realizou-se, no GI, a incisuroplastia troclear, que consistiu no desgaste da porção medial do côndilo lateral do fêmur, com auxílio de fresa óssea acoplada a motor elétrico de baixa rotação, removendose a porção cranial, média e caudal da incisura troclear lateral. Essa manobra foi acompanhada da irrigação intermitente da articulação com solução salina estéril. Foi então confeccionado o retalho de fáscia lata, conforme técnica descrita por COETZEE \& LUBBE (1995). O retalho foi mantido unido à tuberosidade da tíbia, seguido de sua condução craniocaudal por sob o ligamento intermeniscal e, posteriormente, através da articulação com emergência caudal e proximal à fabela lateral. Para fixação do retalho, empregou-se fio de polipropileno $0^{\mathrm{j}}$, com padrão de sutura interrompido simples. A cápsula articular foi suturada com fio de poliglactina 910 2-0 $0^{1}$ com sutura contínua simples, e o defeito na fáscia lata aproximado com sutura interrompida simples com fio de polipropileno 2-0. A porção remanescente do retalho foi suturada sob tensão ao tendão patelar, empregando-se sutura interrompida simples com polipropileno 0. Adermorrafia foi realizada por meio de sutura interrompida simples com náilon 3-0 . No GC, o procedimento cirúrgico foi análogo, exceto pela ausência da incisuroplastia troclear. Para se minimizarem quaisquer efeitos temporais, as cirurgias nas articulações contralaterais foram realizadas em momentos cirúrgicos subseqüentes.

Foi aplicado adesivo de liberação gradativa de fentanil ${ }^{\mathrm{n}}$ à região lombar, imediatamente ao término da cirurgia. A administração de cetoprofeno foi realizada na mesma dose que a utilizada previamente à cirurgia, durante quatro dias subseqüentes ao ato cirúrgico.
Diariamente, efetuou-se limpeza da ferida cirúrgica com solução fisiológica, até o décimo dia pós-operatório, momento da retirada dos pontos.

A presença do movimento de gaveta e o exame radiográfico das articulações foram realizados previamente à cirurgia (T-1), imediatamente após a estabilização articular (T1), e aos 30 (T30), 90 (T90) e 180 (T180) dias pós-operatórios. O movimento de gaveta foi classificado subjetivamente em zero (movimento de gaveta ausente) ou um (movimento de gaveta presente).

O exame radiográfico, em projeção mediolateral com extensão articular total e com flexão de $90^{\circ}$, foi realizado para determinação do CIM e Vv. As projeções foram realizadas com o membro apoiado diretamente sobre o chassi radiográfico a uma distância foco-filme de $100 \mathrm{~cm}$. O plano da flexão articular foi paralelo ao plano do filme, com os raios- $X$ centrados na articulação. O CIM e o Vv resultante foram determinados segundo o método de "Rouleaux" (MITTON et al., 1991), que consistiu na sobreposição dos fêmures nas projeções em flexão e extensão, e determinação de dois pontos de referência idênticos, com intervalo mínimo de distância de quatro a cinco centímetros entre eles. Em seguida, as radiografias foram sobrepostas, de forma que a silhueta da tíbia fosse coincidente, determinando-se o deslocamento dos pontos femorais previamente estabelecidos. $\mathrm{O}$ deslocamento respectivo dos dois pontos demarcados no fêmur foi então unido por uma reta. Posteriormente, determinaram-se as mediatrizes a essas retas, sendo que a intersecção destas evidenciava o CIM. O CIM foi unido por uma reta ao ponto de maior contato articular, evidenciando assim o eixo CIMp. Para determinação do $\mathrm{Vv}$, foi traçada uma reta perpendicularmente à CIMp, conforme sugeriram FRANKEL et al. (1971), PROSTREDNY et al. (1991) e SELMI et al. (2003)

Para avaliação das variáveis nos momentos pós-operatórios, excetuando-se o grau de claudicação, os animais foram tranqüilizados com maleato de acepromazina $1 \%\left(0,1 \mathrm{mg} \mathrm{Kg}^{-1}-\mathrm{IV}\right)$ e, após 15 minutos, anestesiados com tiopental sódico ${ }^{\circ}\left(12,5 \mathrm{mg} \mathrm{Kg}^{-1}-\mathrm{IV}\right)$.

\section{RESULTADOS E DISCUSSÃO}

A análise dos resultados pós-operatórios nas estabilizações cirúrgicas após ruptura do LCCr é muitas vezes subjetiva, baseando-se na avaliação da qualidade da deambulação e presença de instabilidade craniocaudal do joelho (SHIRES \& HULSE, 1984; COETZEE \& LUBBE, 1995; LA PRADE et al., 1998; SELMI et al., 2002) e no uso de radiografias para 
documentação do grau de osteoartrose (SHIRES \& HULSE, 1984; VASSEUR \& BERRY, 1992; COETZEE \& LUBBE, 1995; GEELS et al., 2000). Contudo, variáveis objetivas como perímetro da coxa, amplitude de movimento articular e uso da placa de força (JEVENS et al., 1996; LA PRADE et al., 1998; GEELS et al., 2000) também são recomendadas. A despeito da gama de informações obtidas com o uso da placa de força, a aquisição de dados é dependente de material apropriado, com custo elevado, e muitas vezes com aplicação exclusivamente experimental, sendo restrita a centros de pesquisa. Em contrapartida, a avaliação radiográfica do joelho é de fácil realização e de ampla disponibilidade clínica.

Vários autores têm sugerido o uso de avaliações radiográficas para o diagnóstico da RLCCr (ROOSTER \& VAN BREE, 1999), no entanto, até o momento, as diferentes técnicas radiográficas não foram avaliadas como forma de se estabelecer prognóstico. A documentação radiográfica da progressão de osteoartrose, apesar de amplamente utilizada, não demonstra correlação com a evolução clínica pós-cirúrgica(VASSEUR \& BERRY, 1992; INNES \& BARR, 1998; SELMI et al., 2002) Contrariamente, a determinação radiográfica do CIM tem sido descrita por diversos autores (ARNOCZKY et al., 1977; IRELAND et al., 1986; PROSTREDNY et al., 1991; SELMI et al., 2003) na avaliação pré ou pós-operatória e após estabilizações intra ou extra-articulares.

A avaliação radiográfica pré-operatória dos joelhos dos cães deste estudo evidenciou a presença de CIM extra-articular em todas as articulações, de ambos os grupos, com vetor velocidade tangente ao ponto de contato articular, indicando movimento normal de deslizamento entre as superfícies articulares. O vetor velocidade, que determina as forças compressivas existentes na articulação, é representado por uma reta tangente à superfície articular quando as forças atuantes na articulação apresentam-se neutralizadas, desta forma propiciando menor resistência ao movimento de flexão e extensão do membro (PROSTREDNY et al., 1991). Essa condição é observada quando o CIM encontra-se posicionado na superfície articular no ponto de contato entre as cartilagens articulares, ou quando está localizado de forma extraarticular, perpendicularmente ao ponto de contato articular. Se o CIM localiza-se em uma linha nãoperpendicular ao ponto de maior contato das superfícies articulares, o vetor velocidade naquele ponto não será tangente à superfície condilar, resultando em forças compressivas nas superfícies articulares e conseqüentemente no aumento das forças de fricção durante a movimentação do membro
(FRANKEL et al., 1971; ARNOCZKY et al., 1977; PROSTREDNY et al., 1991).

O CIM descreve objetivamente e de forma reproduzível a estabilidade e o comportamento biomecânico articular (ARNOCSKY et al., 1977; PROSTREDNY et al., 1991) em condições in vivo ou ex vivo (IRELAND et al., 1986). No entanto, é importante ressaltar que o correto posicionamento da articulação, durante exposição radiográfica, é de fundamental necessidade para avaliação do CIM, uma vez que, de acordo com PANJABI, citado por MITTON et al. (1991), o método é sensível a pequenos erros na determinação dos pontos de referência identificados no fêmur. Dentre estes, cita-se que o ângulo formado entre as mediatrizes deve ser diferente de $90^{\circ}$, além de se manter a distância mínima entre os pontos de referência de $5 \mathrm{~cm}$ e o menor deslocamento angular do fêmur entre as posições radiográficas. Ângulos entre as mediatrizes iguais a $90^{\circ}$ são decorrentes de deslocamentos angulares excessivos durante a avaliação do CIM e são responsáveis por variações articulares rotacionais, que dificultam a determinação dos pontos de referência femoral. Cita-se ainda a dificuldade para correta determinação do ponto de maior contato articular radiográfico, visto que se trata de uma superfície de contato e não de um único ponto.

Durante a avaliação física prévia de todos os joelhos estudados, não foi observada a presença de movimento de gaveta ou quaisquer outras alterações músculo-esqueléticas sugestivas de instabilidade articular como excessiva rotação interna da tíbia, crepitação e instabilidade mediolateral. No momento imediatamente após a estabilização articular, três, dentre nove articulações pertencentes ao GC (cães 2, 5 e 8), apresentaram movimento de gaveta. No GI, o movimento de gaveta estava presente em duas articulações durante a extensão (cães 2 e 6). Aos 30 dias de pós-operatório, observou-se presença de movimento de gaveta em duas articulações de GC (cães 2 e 8) e de GI (cães 2 e 6) durante a extensão; aos 90 dias p.o., apenas uma articulação de GC (cão 2) e outra de GI (2) apresentavam instabilidade durante a extensão e, aos 180 dias de avaliação, um animal em cada grupo apresentava instabilidade craniocaudal durante a extensão articular (cão 2 em GC e cão 2 em GI). Apesar da instabilidade clínica observada nos diferentes momentos de avaliação po, todas as articulações apresentavam CIM extra-articular e vetor velocidade normal. Tais observações corroboram a ausência de correlação entre os achados clínicos e avaliações objetivas (SHIRES \& HULSE, 1984; COETZEE \& LUBBE, 1995; LA PRADE et al., 1998; SELMI et al., 2002). As possíveis causas pela presença de 
movimento de gaveta nos cães de ambos os grupos podem ser em decorrência da falta de isometricidade do retalho de fáscia lata ou de tensão inadequada durante a fixação do retalho ao tendão patelar. Vale citar que a instabilidade foi avaliada como presente ou ausente, mas, em nenhum dos joelhos em estudo, a instabilidade foi considerada importante do ponto de vista clínico. AIKEN et al. (1995) e COETZEE \& LUBBE (1995) descrevem instabilidade residual em seus estudos, e a atribuem à falta de tendão do retalho articular durante a fixação. Os dados referentes ao número de animais com movimento de gaveta e à localização do CIM e do vetor velocidade estão dispostos na tabela 1 .

Neste estudo, visto a natureza da avaliação pós-operatória, não foi possível determinar o CIM e o Vv após transecção do LCCr, uma vez que, para tal, seria necessário suturar a ferida cirúrgica, de modo a restabelecer a integridade dos mecanismos secundários de estabilidade articular, evitando a influência deste fator na determinação do CIM. Deste modo, tornou-se impraticável avaliar se os animais deste estudo apresentaram ou não alterações do CIM e do Vv após transecção do LCCr imediatamente antes do reparo cirúrgico. A estabilização articular proposta por COETZEE \& LUBBE (1995), utilizada neste estudo, é derivada da técnica “over-the-top” sugerida por ARNOCZKY et al. (1977). Estes autores avaliaram o CIM e o Vv resultante após estabilização intra-articular em cadáveres caninos, e relataram a manutenção do CIM, e respectivo Vv, antes da transecção do LCCr, após a mesma, e imediatamente após a estabilização cirúrgica. Contrariamente, MITTON et al. (1991), utilizando a mesma técnica em casos clínicos, referiram diferenças significativas entre o CIM de joelhos estáveis e instáveis, e atribuíram tais diferenças ao estresse radiográfico imposto à articulação e a possíveis lesões meniscais diagnosticadas durante o ato cirúrgico.
SELMI et al. (2003) relataram similaridade aos achados de MITTON et al. (1991); entretanto, mesmo sem terem utilizado exposição radiográfica com as articulações em estresse e sem descreverem lesões de menisco, sugeriram o estresse articular de forma a evidenciar possíveis alterações do CIM. Discrepâncias entre os achados em articulações estáveis e instáveis foram observadas por PROSTREDNY et al. (1991), embora as possíveis causas destas diferenças não serem conhecidas.

Em pacientes com LCCr, a articulação do joelho apresenta movimento de rolagem e de deslizamento que podem ser confirmados pela posição intra ou extra-articular do CIM, respectivamente (ARNOCZKY et al., 1977; IRELAND et al., 1986; PROSTREDNY et al., 1991). Biomecanicamente, o CIM encontrava-se em posição extra-articular em todas as articulações de ambos os grupos, acompanhado de $\mathrm{Vv}$ tangente à superfície articular, indicando a manutenção do movimento de deslizamento observado no momento pré-operatório. Este comportamento foi observado até 180 dias de avaliação po, e evidencia a eficácia clínica da técnica proposta por COETZEE \& LUBBE (1995), nos joelhos do grupo controle e naqueles submetidos a ITR. Estas observações são corroboradas pelas de LA PRADE et al. (1998), que relataram manutenção da marcha após ITR em joelhos caninos estáveis. Contudo, JEVENS et al. (1996) e GEELS et al. (2000) descreveram a diminuição do apoio após estabilização articular utilizando a técnica "under-and-over" proposta por SHIRES \& HULSE (1984). Há de se considerar que a avaliação do CIM e do Vv são estáticas e refletem o comportamento das superfícies articulares numa dada variação de amplitude de movimento articular, enquanto que análises por placa de força demonstram a cinética do movimento articular (JEVENS et al., 1996). Entretanto, a análise isolada do CIM não indica se ocorre compressão ou separação das superfícies

Tabela 1 - Freqüência do movimento de gaveta, centros instantâneos de movimento (CIM) e vetores velocidade (Vv), após reconstrução articular com retalho de fáscia lata, precedida (GI) ou não de incisuroplastia troclear (GC) em cães submetidos à transecção do ligamento cruzado cranial.

\begin{tabular}{|c|c|c|c|c|c|c|}
\hline \multirow{2}{*}{ Grupo } & \multirow{2}{*}{ Variável } & \multicolumn{5}{|c|}{ Tempo de avaliação } \\
\hline & & $\mathrm{T}-1$ & $\mathrm{~T} 1$ & Т30 & T90 & T180 \\
\hline \multirow{3}{*}{ GC } & Movimento de gaveta (extensão) & - & $+(3)$ & $+(2)$ & $+(1)$ & $+(1)$ \\
\hline & CIM & $\mathrm{E}$ & $\mathrm{E}$ & $\mathrm{E}$ & $\mathrm{E}$ & E \\
\hline & Vv & $\mathrm{N}$ & $\mathrm{N}$ & $\mathrm{N}$ & $\mathrm{N}$ & $\mathrm{N}$ \\
\hline \multirow{3}{*}{ GI } & Movimento de gaveta (extensão) & - & $+(2)$ & $+(2)$ & $+(1)$ & $+(1)$ \\
\hline & CIM & $\mathrm{E}$ & $\mathrm{E}$ & E & E & $\mathrm{E}$ \\
\hline & $\mathrm{Vv}$ & $\mathrm{N}$ & $\mathrm{N}$ & $\mathrm{N}$ & $\mathrm{N}$ & $\mathrm{N}$ \\
\hline
\end{tabular}

O dígito entre parênteses representa o número de animais com presença de alterações. + - presença de movimento de gaveta. - - ausência de movimento de gaveta. E - localização extra-articular, $\mathrm{N}$ - vetor velocidade tangente à superfície articular. 
cartilaginosas durante o movimento articular. Quando essa variável é analisada juntamente com o sentido do Vv, pode-se determinar se existem ou não forças compressivas ou de distração que imponham resistência ao movimento articular. Pela manutenção do Vv tangente à superfície articular, acredita-se que a biomecânica dos joelhos, após estabilização cirúrgica associada ou não a ITR, foi preservada.

Apesar de os efeitos biomecânicos não terem sido alterados durante o período de avaliação nas articulações deste estudo, deve-se considerar que o método utilizado negligencia os efeitos rotacionais da tíbia durante a flexão articular. De acordo com MITTON et al. (1991), o CIM e o Vv resultante são determinados principalmente em articulações planares, ou seja, articulações que, quando observadas perpendicularmente a um plano, apresentam velocidade resultante igual a zero num determinado ponto, a cada instante do movimento. Desta forma, considera-se o joelho como uma articulação planar em quase sua totalidade de amplitude.

Relativamente à presença de movimento de gaveta durante a extensão articular, observou-se comportamento similar entre os grupos. Considerandose a subjetividade da avaliação do movimento de gaveta, e a falta de correlação deste parâmetro com a função clínica (INNES \& BARR, 1998), pode-se afirmar que, a despeito deste achado de forma regressiva em ambos os grupos, as articulações apresentavam-se dentro da normalidade, fato confirmado pelas observações referentes ao CIM e ao Vv.

\section{CONCLUSÕES}

Conclui-se que a estabilização articular com retalho de fáscia lata associada ou não a incisuroplastia troclear não altera o posicionamento do centro instantâneo de movimento ou o vetor velocidade resultante, mantendo a integridade biomecânica articular, apesar da presença regressiva de movimento de gaveta.

\section{FONTES DE AQUISIÇÃO}

${ }^{a}$ Golden Formula, Grandfood do Brasil. SP.

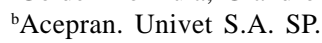

'Propoabbott. Abbott do Brasil. SP.

${ }^{\mathrm{d}}$ Ketofen. Merial. SP.

eDimorf. Cristália. SP.

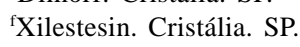

'Bupivacaína. Cristália. SP.

hevorane. Abbott do Brasil. SP.

${ }^{\mathrm{i} C e f a l e x i n a . ~ A r i s t o n . ~ S P . ~}$

jProlene. Ethicon. SP.

${ }^{1}$ Vicryl. Ethicon. SP.

${ }^{\mathrm{m}}$ Ethilon. Ethicon. SP

mDurogesic. Jansen \& Cillag. Canadá.

${ }^{\circ}$ Thionembutal. Abbott do Brasil. SP

\section{COMISSÃO DE ÉTICA E BIOSSEGURANÇA}

O trabalho foi submetido e aprovado pela Comissão de Ética da Universidade de Brasília, e realizado de acordo com as normas do COBEA.

\section{REFERÊNCIAS}

AIKEN, S.W. et al. Intercondylar notch width in dogs with and without cranial cruciate ligament injuries. Veterinary and Comparative Orthopedics and Traumatology, Berlim, v.8, p.128-132, 1995.

ARNOCZKY, S.P. et al. Biomechanical evaluation of anterior cruciate ligament repair in the dog: an analysis of the instant center of motion. Journal of the American Animal Hospital Association, Lakewood, v.13, p.553-558, 1977.

COETZEE, G.L.; LUBBE, A.M. A prospective study comparing two fascial reconstruction techniques to stabilize the cranial cruciate deficient stifle in the dog. Veterinary and Comparative Orthopedics and Traumatology, Berlim, v.8, p.82-90, 1995.

ELKINS, A.D. et al. A retrospective study evaluating the degree of degenerative joint disease in the stifle joint of dogs following surgical repair of anterior cruciate ligament rupture. Journal of the American Animal Hospital Association, Lakewood, v.27, p.533-540, 1991.

FITCH, R.B. et al. The intercondylar fossa of the normal canine stifle: an anatomic and radiographic study. Veterinary Surgery, Santa Barbara, v.24, p.148-155, 1995a.

FITCH, R.B. et al. The effect of intercondylar notchplasty on the normal canine stifle. Veterinary Surgery, Santa Barbara, v.24, p.156-164, 1995b.

FRANKEL, V.H. et al. Biomechanics of internal derangement of the knee. Pathomechanics as determined by analysis of the ICM. Journal of Bone and Joint Surgery, Sacramento, v.53A, p.945-962, 1971.

GEELS, J.J. et al. Evaluation of an intracapsular technique for the treatment of cranial cruciate ligament rupture. Veterinary and Comparative Orthopedics and Traumatology, Berlim, v.13, p.197-203, 2000.

INNES, J.F.; BARR, A.R.S. Clinical natural history of the postsurgical cruciate deficient canine stifle joint: 1 year. Journal of Small Animal Practice, Londres, v.39, p.325-332, 1998.

IRELAND, W.P. et al. Location of the instantaneous center of rotation in the normal canine stifle. American Journal of Veterinary Research, Schaumburg, v.47, p.837-840, 1986.

JEVENS, D.J. et al. Use of force-plate analysis of gait to compare two surgical techniques for treatment of cranial cruciate ligament rupture in dogs. American Journal of Veterinary Research, Schaumburg, v.57, n.3, p.389-393, 1996.

LA PRADE, R.F. et al. The effects of aggressive notchplasty on the normal knee in dogs. American Journal of Sports Medicine, Detroit, v.26, n.2, p.193-200, 1998.

MANN, T.A. et al. The natural history of the intercondylar notch after notchplasty. American Journal of Sports Medicine, Detroit, v.27, n.2, p.181-188, 1999. 
MITTON, G.R. et al. Evaluation of the instantaneous center of rotation of the stifle before and after repair of torn cruciate ligament by use of the over-the-top technique in dogs. American Journal of Veterinary Research, Schaumburg, v.52, n.10, p.1731-1737, 1991.

PROSTREDNY, J.M. et al. Effect of suture type on stifle joint biomechanics after extra-articular repair of cranial cruciate ligament transection in the dog. Veterinary and Comparative Orthopedics and Traumatology, Berlim, v.4, p.144-149, 1991.

ROOSTER, H.; VAN BREE, H. Radiographic measurement of craniocaudal instability in the stifle joints of clinically normal dogs and dogs with injury of a cranial cruciate ligament. American Journal of Veterinary Research, Schaumburg, v.60, n.12, p.1567-1570, 1999.

SELMI, A.L. et al. Clinical radiographic evaluation of a polyester prosthesis in dogs with cranial cruciate ligament rupture. Ciência Rural. Santa Maria, v.32, n.5, p.793-798, 2002.
SELMI, A.L. et al. Centro instantâneo de movimento, na avaliação ex-vivo da reconstrução extra-articular fabelo-tibial, após transecção do ligamento cruzado cranial em cães. Ciência Rural. Santa Maria, v.33, n.5, p.875-880, 2003.

SHIRES, P.K.; HULSE, D.A. The under-and-over fascial replacement technique for anterior cruciate ligament rupture in dogs: a retrospective study. Journal of the American Animal Hospital Association, Lakewood, v.20, p.69-77, 1984.

VASSEUR, P.B.; BERRY, C.R. Progression of stifle osteoarthrosis following reconstruction of the cranial cruciate ligament in 21 dogs. Journal of the American Animal Hospital Association, Lakewood, v.28, p.129-136, 1992.

VASSEUR, P.B. et al. Evaluation of the Leeds-Keio synthetic replacement for the cranial cruciate ligament in dogs: an experimental study. Veterinary and Comparative Orthopedics and Traumatology, Berlim, v.9, p.66-74, 1996. 